OPEN ACCESS

Edited by:

Daniel E. Voth,

University of Arkansas for Medical

Sciences, United States

Reviewed by:

Andréa Cristina Fogaça,

University of São Paulo, Brazi Isaura Simões,

University of Coimbra, Portugal

*Correspondence:

Xin-Ru Wang

wang8848@umn.edu

Benjamin Cull

cull0122@umn.edu

Specialty section: This article was submitted to

Bacteria and Host,

a section of the journal

Frontiers in Cellular and

Infection Microbiology

Received: 27 September 2021 Accepted: 11 January 2022

Published: 27 January 2022

Citation:

Wang X-R and Cull B (2022) Apoptosis and Autophagy: Current Understanding in Tick-

Pathogen Interactions.

Front. Cell. Infect. Microbiol. 12:784430. do: $10.3389 /$ fcimb.2022.784430

\section{Apoptosis and Autophagy: Current Understanding in Tick-Pathogen Interactions}

\author{
Xin-Ru Wang ${ }^{*}$ and Benjamin Cull ${ }^{*}$ \\ Department of Entomology, University of Minnesota, St. Paul, MN, United States
}

Tick-borne diseases are a significant threat to human and animal health throughout the world. How tick-borne pathogens successfully infect and disseminate in both their vertebrate and invertebrate hosts is only partially understood. Pathogens have evolved several mechanisms to combat host defense systems, and to avoid and modulate host immunity during infection, therefore benefitting their survival and replication. In the host, pathogens trigger responses from innate and adaptive immune systems that recognize and eliminate invaders. Two important innate defenses against pathogens are the programmed cell death pathways of apoptosis and autophagy. This Mini Review surveys the current knowledge of apoptosis and autophagy pathways in tick-pathogen interactions, as well as the strategies evolved by pathogens for their benefit. We then assess the limitations to studying both pathways and discuss their participation in the network of the tick immune system, before highlighting future perspectives in this field. The knowledge gained would significantly enhance our understanding of the defense responses in vector ticks that regulate pathogen infection and burden, and form the foundation for future research to identify novel approaches to the control of tickborne diseases.

Keywords: tick, intracellular pathogens, apoptosis, autophagy, cross-talk, Rickettsia, Anaplasma, Ehrlichia

\section{INTRODUCTION}

Ticks, as obligate blood-sucking arthropods, can cause substantial public health burdens by direct feeding behaviors and transmitting a broad range of viral, bacterial, and protozoan pathogens to hosts. To date, approximately 80 known tick species are recognized as vectors responsible for spreading emerging infectious diseases throughout the world (Figure 1) (Jongejan and Uilenberg, 2004; Ghosh et al., 2007). For example, in the USA, tick-borne diseases (TBDs) accounted for nearly $76.5 \%$ of all vector-borne diseases from 2004 to 2016, based on the Centers for Disease Control and Prevention reports (CDC, 2018). Additionally, the effects of human activities and climate change on tick distribution and abundance also increase the risk of emerging and re-emerging diseases (Gray and Banerjee, 1999; Gray et al., 2009). The initial step for the success of intracellular pathogens is survival within their hosts. Tick-borne obligate intracellular pathogens include arboviruses and bacteria, which are responsible for diseases of medical and veterinary importance globally. These pathogens use different strategies to survive within their hosts. For example, after entry into the host cell Rickettsia spp. escape the endo-lysosomal pathway and replicate in the cytosol, whilst 


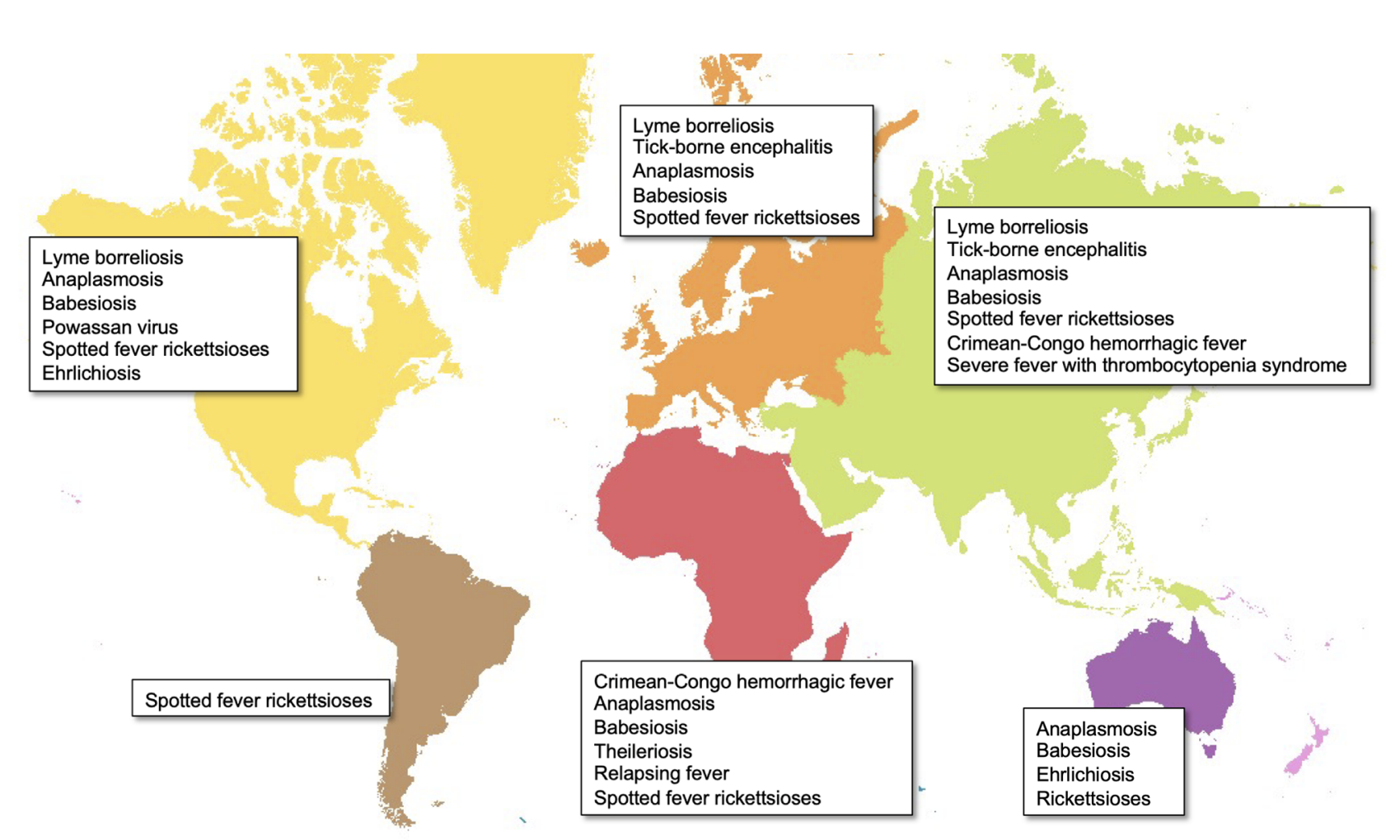

FIGURE 1 | Global distribution of major tick-borne diseases by continent. Map created in ArcGIS online (ESRI, California) using open-source layers from Natural Earth (naturalearthdata.com).

Anaplasma and Ehrlichia survive and replicate within specialized vacuoles (Salje, 2021). Considering these diseases are maintained in nature by cycling between ticks and their mammalian hosts, understanding tick-pathogen interactions provides clues for pathogen transmission and establishes a foundation for developing preventative strategies against human infection.

Programmed cell death (PCD) is an essential process in eukaryote homeostasis and development, and includes multiple death programs, such as apoptosis and autophagy (Ameisen, 2002). As a type I PCD mechanism, apoptosis is a genetically regulated process of cellular suicide in multicellular organisms (Taatjes et al., 2008). As a type II PCD mechanism, autophagy is a highly conserved cellular recycling process characterized by lysosomal degradation of cytosol and organelles and recycling of the breakdown products (Levine and Deretic, 2007). Commonly, PCD has been studied in the context of a broad range of human diseases, including neurodegenerative disorders, cancer, and ischemic damage (Elmore, 2007). Recently, different types of PCD have been identified as critical components of innate immunity pathways that act as defense mechanisms against intracellular bacteria, parasites, and viruses, even in insects (Fuchs and Steller, 2011; Romanelli et al., 2014; Koonin and Krupovic, 2019). Like all invertebrates, ticks lack an adaptive immune system but are capable of using the innate immune system to regulate pathogen colonization, persistence, and transmission (Brossard and Wikel, 2004; Hart and Thangamani, 2021). This Mini Review brings together current knowledge on the apoptosis and autophagy pathways from the perspective of tick-pathogen interactions, and our progress in deciphering the mechanisms used by tick-borne pathogens to interact with these pathways. The crosstalk between apoptosis, autophagy and other immune pathways, and how this might be interfered with by intracellular pathogens to benefit their survival are also investigated. Finally, we discuss challenges in studying these pathways in tick-pathogen systems and how these may be overcome in future to improve our understanding of this field and its potential importance to pathogen persistence in ticks and vector competence.

\section{APOPTOSIS}

As a genetically regulated process of cellular suicide in multicellular organisms, apoptosis responsible for development and homeostasis has been described in several models, including in some arthropods (Jacobson et al., 1997; Bowman and Sauer, 2004; Taatjes et al., 2008; Menze et al., 2010). In vertebrates, apoptosis is also recognized as an innate immune pathway, and mediates eukaryotic cell response to infection by a wide range of pathogens. However, the roles of apoptosis are still perplexing and complex, with multiple pro- and anti-death factors underlying the diversity of events (Everett and McFadden, 1999; Rudel et al., 2010). In some cases, activation of apoptosis is destructive for the pathogens. Pathogens are internalized and 
packed into the apoptosome during infection, resulting in a more efficient fusion of the phagosome/lysosome, followed by digestion and degradation. Upon apoptosis activation, upregulating nucleases and enzymes can also result in cell demise and promote pathogen clearance (Cervantes et al., 2007; Willingham et al., 2007; Taylor et al., 2008). In addition, efferocytosis, as an antimicrobial effect of apoptosis, allows rapid bacterial killing (Martin et al., 2012; Behar and Briken, 2019). On the other hand, apoptosis can also be advantageous for pathogens. For example, infected apoptotic cells may become unable to contain pathogens and cease to function as a barrier, thus promoting their spread to neighboring cells. Also, in some studies it was shown that induction of apoptosis protected the pathogens against phagocytosis and innate host defenses, which was beneficial for pathogen survival (Green and Kroemer, 2004; Riedl and Salvesen, 2007). Another prominent defense strategy of the host is to eliminate the replicative niche of pathogens via the destruction of infected tissues, thereby preventing their replication and dissemination (Labbé and Saleh, 2008). Obviously, the relationships between host and pathogens are complex and involve an intricate balance to serve both host and pathogen interests. This balance of pathogen-induced apoptosis depends on the taxa of bacteria, the duration of infection, the host cell type, multiplicity of infection (MOI), and other factors.

Apoptosis is important for tick development as it initiates salivary gland degeneration, which is regulated by a cascade of caspases leading to the degradation of DNA and proteins in acini (Mao et al., 1995; Mao and Kaufman, 1999; L’Amoreaux et al., 2003; Nunes et al., 2005; Scopinho Furquim et al., 2008). Similar to vertebrates, the regulation of apoptosis in some arthropods and the interactions with pathogens they harbor and transmit have been described (Clarke and Clem, 2003; Flegel, 2007; Sokolova, 2009). However, a few mechanistic insights are just beginning to be revealed in this field due to the wide range of pathogens and their various arthropod hosts. Here, we focus on tick-borne obligate intracellular pathogens to illustrate the paradigms of the function of the apoptotic machinery in ticks.

\section{Inhibition of Host Apoptosis by Tick-Borne Intracellular Pathogens}

A prime example of intracellular bacteria regulating apoptosis is Anaplasma phagocytophilum, a tick-transmitted rickettsial agent that causes human granulocytic anaplasmosis. In vitro, A. phagocytophilum utilizes several mechanisms to inhibit apoptosis in different species of tick cells. For example, besides interfering with endoplasmic reticulum (ER) and the unfolded protein response in Ixodes scapularis ISE6 cells, A. phagocytophilum also downregulates expression of a series of kinases, including phosphoenolpyruvate carboxykinase (PEPCK), mitogenactivated protein kinase (MKK), and apoptosis signal-regulating kinase 1 (ASK1) (Ayllón et al., 2013; Villar et al., 2015). In addition, in $I$. ricinus IRE/CTVM20 tick cells, transcriptome analysis and flow cytometry revealed that infection with $A$. phagocytophilum not only regulates JAK and anti-apoptotic factors gene expression but also inhibits the intrinsic apoptosis pathway (Alberdi et al., 2016b; Alberdi et al., 2016a). In vivo, A. phagocytophilum displays a tissue-specific response to mediate cell apoptosis in tick nymphs (Ayllón et al., 2015). It achieves this by targeting the JAK/STAT pathway and decreasing FAS expression in midguts, while reducing porin (voltage-dependent anionselective channel) expression to inhibit cytochrome $\mathrm{c}$ (one of the mitochondrial proteins associated with apoptosis) release from mitochondria in salivary glands via intrinsic apoptosis. Interestingly, tick salivary glands serve as an essential organ for A. phagocytophilum colonization, and have a possible role in activating extrinsic apoptosis to limit bacterial infection. Indeed, mitochondria, caspases, and pro/anti-apoptotic molecules are key players associated with apoptosis during pathogen infection. Similar to its manipulation of the arthropod host, $A$. phagocytophilum also modulates activity of the above "players" to inhibit apoptosis for their advantage in vertebrate hosts (Scaife et al., 2003; Borjesson et al., 2005; Choi et al., 2005; Ge and Rikihisa, 2006; Niu et al., 2010).

Another very similar system is exploited by Rickettsia rickettsii (agent of Rocky Mountain spotted fever). According to the proteome of Rhipicephalus microplus BME26 cells, $R$. rickettsii may hamper apoptosis via inhibition of caspase-3 activity, thus favoring bacterial growth and proliferation (Martins et al., 2020). Apart from bacterial pathogens, tick-borne viruses have long received much attention. The $I$. ricinus IRE/CTVM20 tick cell transcriptome exerts different gene expression patterns relative to infection with $A$. phagocytophilum after tick-borne encephalitis virus (TBEV) and louping ill virus (LIV) infection, such as raising cytochrome c expression. Interestingly, some apoptosis-related genes, including caspase and hsp70, are expressed differently in flavivirus and intracellular bacterial infections. Whether flaviviruses could benefit from inhibiting tick apoptosis as does $A$. phagocytophilum, is however unconfirmed (Mansfield et al., 2017).

\section{Activation of Host Apoptosis by Tick- Borne Intracellular Pathogens}

Although a critical strategy of intracellular pathogens is induction of vertebrate host-cell apoptosis under certain circumstances, the pro-apoptotic response of ticks to tick-borne pathogens is much less defined. Recently, we have shown that Rickettsia parkeri, a tick-transmitted spotted fever group rickettsia, is able to activate mitochondria-dependent apoptosis to promote its infection of and replication in tick cells (Wang X-R. et al., 2021). By employing cell types from different tick species, we demonstrated that $R$. parkeri initiation of apoptosis was a conserved response and required intracellular rickettsial replication. Considering that apoptosis is initiated via a series of stressors within a cell, the different growth status (exposure, colonization, invasion, and infection) of pathogens in the host might cause different stimuli, thus playing a diverse role in apoptosis. Indeed, $R$. parkeri exerts pro-and antiapoptotic activities during different infection phases, which also has been observed in another spotted fever group rickettsia, $R$. rickettsii (Clifton et al., 1998). However, unlike in the arthropod host, $R$. parkeri failed to induce apoptosis in vertebrate host cells at the same infection phase (Wang X-R. et al., 2021). Unsurprisingly, pro-apoptotic activity in one cell type or host species may not be the same as in another cell type or host species. Another example of a pathogen that induces different responses in mammalian and tick hosts is Hazara virus, a tick-borne segmented negative-sense 
RNA virus closely related to Crimean-Congo hemorrhagic fever virus (CCHFV), but which causes less severe disease. Cleavage of virus nucleocapsid (N) protein by caspase-3 results in apoptosis in mammalian cells but fails to activate apoptosis in tick cells. The use of two different strategies to modulate apoptosis in the respective hosts by members of the genus Nairovirus could directly affect the infection outcome (Fuller et al., 2019). Obviously, a flexible strategy is important for the successful colonization of vectors and hosts by those pathogens in a broad range of host cell types and host species. However, how intracellular pathogens execute this flexible strategy is largely unexplored in their arthropod hosts.

\section{AUTOPHAGY}

Autophagy is an important eukaryotic homeostatic pathway involved in the recycling of intracellular constituents and survival during starvation. Although various subtypes of autophagy exist, the best understood is macroautophagy (often simply called autophagy), characterized by formation of a double membrane-bound vesicle called an autophagosome and subsequent trafficking of the autophagosome to the lysosome for breakdown of its contents (Feng et al., 2014). Compared to our rapidly advancing knowledge of autophagy in vertebrates, that of autophagic processes in ticks is still in its infancy, with the core autophagic machinery only identified in a few species (Umemiya et al., 2007; Kawano et al., 2011; Flores Fernández et al., 2014; Umemiya-Shirafuji et al., 2014; Flores Fernández et al., 2016; Moura-Martiniano et al., 2017; Wang et al., 2020). As well as its important role in starvation (Umemiya-Shirafuji et al., 2010; Umemiya-Shirafuji et al., 2014; Moura-Martiniano et al., 2017; González Castillo et al., 2019; Rosendale et al., 2019; Wang et al., 2020), autophagy in ticks is also involved in embryo development (Umemiya-Shirafuji et al., 2010; Kawano et al., 2011; Umemiya-Shirafuji et al., 2014; Flores Fernández et al., 2014; González Castillo et al., 2019) and degeneration of salivary glands (Yu et al., 2017; Wang et al., 2018; Wang Y. et al., 2021), in which apoptosis also plays a role. Despite autophagy being a key component of host innate immune response to pathogens [known as xenophagy (Wang and Li, 2020)], the function and mechanism of autophagy in the interactions between ticks and tick-borne pathogens is completely unknown. Considering that autophagy is a highly conserved process, the common strategies employed by tick-borne pathogens in vertebrates, such as manipulating the host autophagic machinery to evade engulfment and destruction in the lysosome, and/or to direct autophagic processes, may also apply in ticks. Here, we center on the Rickettsiales (Patterson et al., 2021; Salje, 2021; Voss and Rahman, 2021), to display the autophagy mechanisms exploited by tick-borne intracellular bacteria.

\section{Subversion/Evasion of Host Autophagy by Tick-Borne Intracellular Bacteria}

The most well-studied mechanisms used by tick-borne bacteria to manipulate the autophagic pathway come from the
Anaplasmataceae in their interactions with mammalian cells. Anaplasma phagocytophilum secretes the effector protein Ats-1, which interacts with Beclin1 to recruit autophagosomes to the $A$. phagocytophilum vacuole, supplying nutrients to support pathogen growth (Niu et al., 2012). A similar but distinct process to induce autophagy and trafficking of autophagosomes to the pathogen-containing vacuole is employed by Ehrlichia chaffeensis (causative agent of human ehrlichiosis) via its effector Etf-1, which interacts with Rab5, Beclin1 and the autophagyinitiating class III phosphatidylinositol 3-kinase complex (Lin et al., 2016). Both A. phagocytophilum and E. chaffeensis also prevent their vacuoles fusing with the lysosome (Niu et al., 2008; Lin et al., 2016; Lina et al., 2017); this is achieved by E. chaffeensis through modulation of the Wnt signaling pathway to inhibit autolysosome formation (Lina et al., 2017).

Infection with a range of spotted fever group rickettsiae $(R$. conorii, $R$. japonica, $R$. montanensis, $R$. parkeri and $R$. rickettsii) results in autophagy induction in mammalian host cells (Uchiyama et al., 2012; Engström et al., 2019; Sahni et al., 2020). Pathogenic rickettsiae appear to be capable of evading this immune response, whilst non-pathogenic species lack this ability (Uchiyama et al., 2012; Engström et al., 2019). Infection of human umbilical vein endothelial cells with $R$. rickettsii or $R$. conorii results in mTOR activation, potentially as a mechanism by which these rickettsiae limit anti-microbial autophagy (Sahni et al., 2020), whilst Rickettsia parkeri is able to evade autophagy by employing outer membrane protein $\mathrm{B}(\mathrm{OmpB})$ to prevent the ubiquitination of surface proteins and their subsequent recognition by autophagic receptors in both human microvascular endothelial cells and mouse bone-marrowderived macrophages (Engström et al., 2019). Even in the same cell type, different Rickettsia species utilize contrasting strategies to evade autophagy. For example, $R$. australis induces autophagy to aid successful invasion of mouse bone-marrow-derived macrophages (Bechelli et al., 2019), resulting in the inhibition of inflammatory cytokine secretion to favor bacterial survival (Bechelli et al., 2021). Due to the broad range of rickettsial pathogens and different host cell types studied, we are just unveiling the tip of the iceberg regarding the complex interactions between these pathogens and their hosts. Although we can use the situation in vertebrates as a basis for predicting what might occur in ticks, we cannot assume that the strategies employed by pathogens to infect mammals can be generalized to their persistence in arthropods (as shown above with apoptosis), and so further investigation into how pathogens interact with tick autophagy are warranted.

\section{DISCUSSION}

\section{Limitations of Apoptosis and Autophagy Study in Tick-Pathogen Interactions}

Despite impressive progress being made on how pathogens "tamper with" the tick immune system, including revealing antibacterial and antiviral pathways and identifying molecular effectors and cells (Fogaça et al., 2021), unlike the well-known arthropod-pathogen systems (Buchon et al., 2014), tick- 
pathogen interaction is a newly emerging field that is far from completely understood. This is largely due to the tick's own complex development and the diversity of its transmitted pathogens. Firstly, in vitro study depends on tick cell lines, which are relatively fragile compared to more common cell types, and have more intensive culture requirements (Munderloh and Kurtti, 1989; Bell-Sakyi, 1991; Mattila et al., 2007; Bell-Sakyi et al., 2009). Additionally, most tick cell lines were derived from embryos, however, their tissue(s) of origin are unconfirmed. Different cell types might possess different characteristics and ontogenies, resulting in unique properties (Mattila et al., 2007; Wang et al., 2020). In vivo studies on ticks are more challenging because of their unique life cycle, which is influenced by an array of elements, including species, host feeding preference, different ecological and geographic factors in nature, and strict maintenance requirements in the laboratory (Sonenshine and Roe, 2013; Jia et al., 2020). Besides some medically and veterinary important species, most ticks are a blind-spot due to insufficient data on genomic information, let alone the interactions with potential pathogens that they may harbor. Genomic data is only available for a limited number of tick species including Ixodes scapularis, Ixodes ricinus, Ixodes persulcatus, Haemaphysalis longicornis, Dermacentor silvarum, Hyalomma asiaticum, Rhipicephalus sanguineus, and Rhipicephalus microplus (Gulia-Nuss et al., 2016; Cramaro et al., 2017; Jia et al., 2020). The most critical difficulty is pathogens themselves, as many different factors such as species/strain pathogenicity, difficulty in laboratory maintenance, etc. may alter the observed results. For example, even different strains of the same pathogen, $R$. rickettsii, exhibits exclusive manners in different cell types or organs (Lehman et al., 2018). Thus, pathogens initiate a critical step for tick immune response, directing more complex communication than simple inhibition or activation. Studies to address these difficulties would pave the way for future research centered on the tick immune system (Figure 2).

\section{Future Perspectives of Apoptosis and Autophagy Study in Tick-Pathogens Interaction}

It is unquestionable that PCD acts as one piece of the puzzle for tick innate immunity, and more work needs to be done to gain more clues to solve a tick's "Jigsaw puzzles". This would include investigating the network of other immune pathways as well as the cross-talk between apoptosis and autophagy under certain conditions (Fairlie et al., 2020). Paradigms in vertebrate hosts utilize these communications to enhance the recognition and destruction of intracellular pathogens (Hua et al., 2019; Van Opdenbosch and Lamkanfi, 2019), and we expect that immune responses in ticks behave similarly. Interestingly, tickborne intracellular pathogens also make use of effective communications in their vertebrate hosts to tip the scales in their favor. For example, as well as inducing autophagy, both the A. phagocytophilum effector Ats-1 and the E. chaffeensis Etf-1 are also translocated into the host mitochondria to inhibit apoptosis initiation (Niu et al., 2010; Liu et al., 2012). Another A. phagocytophilum effector AptA induces autophagy and the ubiquitin-proteasome system, whilst reducing the efficiency of apoptosis (Ma et al., 2021). There is also interplay between autophagy and inflammatory pathways during both Ehrlichia and Rickettsia infection (Tominello et al., 2019; Bechelli et al., 2021), and autophagy induction is balanced by signaling of MyD88 (a downstream adaptor for many pattern recognition receptors) during ehrlichial infection (Kader et al., 2017).

As with other arthropods, crosstalk within the tick innate immune system associated with the response to pathogen infection has also been explored in recent decades (CapelliPeixoto et al., 2017; Fogaça et al., 2021). Although antibacterial and antiviral pathways, including JAK-STAT (Janus kinase/signal transducer and activator of transcription), Toll, IMD (Immune Deficiency) and RNA interference (RNAi), possess a certain specificity, they are also capable of collaboration under certain conditions. For example, ticks utilize the IMD pathway in response to infection with $B$. burgdorferi, A. phagocytophilum, or A. marginale (Shaw et al., 2017; McClure Carroll et al., 2019; Kurokawa et al., 2020). The downstream pathways include NF-kB/ Relish and Jun N-terminal kinase (JNK) (Ramphul et al., 2015; Chowdhury et al., 2020; Tafesh-Edwards and Eleftherianos, 2020), which are involved in viral-induced apoptosis and have been well characterized in insects. It is possible that bacteria induce a similar response in ticks. However, the components of these pathways in ticks are highly divergent from vertebrate and insect systems, and whether those mechanisms also apply in ticks needs further investigation. Towards this end, identification of the apoptosis and autophagy components of ticks by employing comparative genomics would be a significant step. Utilizing tick and other arthropod genome sequences, the homologues to apoptosis/ autophagy-related genes of known function should be confirmed (Wang et al., 2020).

To understand the biological processes involved in apoptosis/ autophagy in response to pathogens, multi-omics (including genetics, epigenetics, transcriptomics, proteomics, metabolomics, and cellomics) would be valuable approaches (Hasin et al., 2017; Chu et al., 2021). For example, combining genomic and transcriptome data can reveal apoptosis-related genes and their roles in pathogen infection. Using metabolomics and proteomics, proteins involved in the apoptosis pathway and their connection to molecular changes in metabolic pathways can also be identified. Single-cell/nucleus omics also can determine functional molecules of each cell and specific tick cell subtypes in response to pathogens. Finally, combining properly analyzed approaches and apoptosis assay measurements, the study of apoptosis in tick-pathogen interactions would be significantly enhanced. Genetic tools for editing microorganisms, including mutagenesis and CRISPR as well as RNAi, facilitate the growing body of research in hostpathogen interactions (Durvasula et al., 1997; Billmyre et al., 2013; Wilke and Marrelli, 2015; Vo et al., 2021). As well as using the multi-omics methods outlined above, further work to characterize interactions between pathogens and tick autophagy could employ transgenic reporter bacteria or viruses to visualize responses to autophagy. In addition, with the use of directed mutants or 


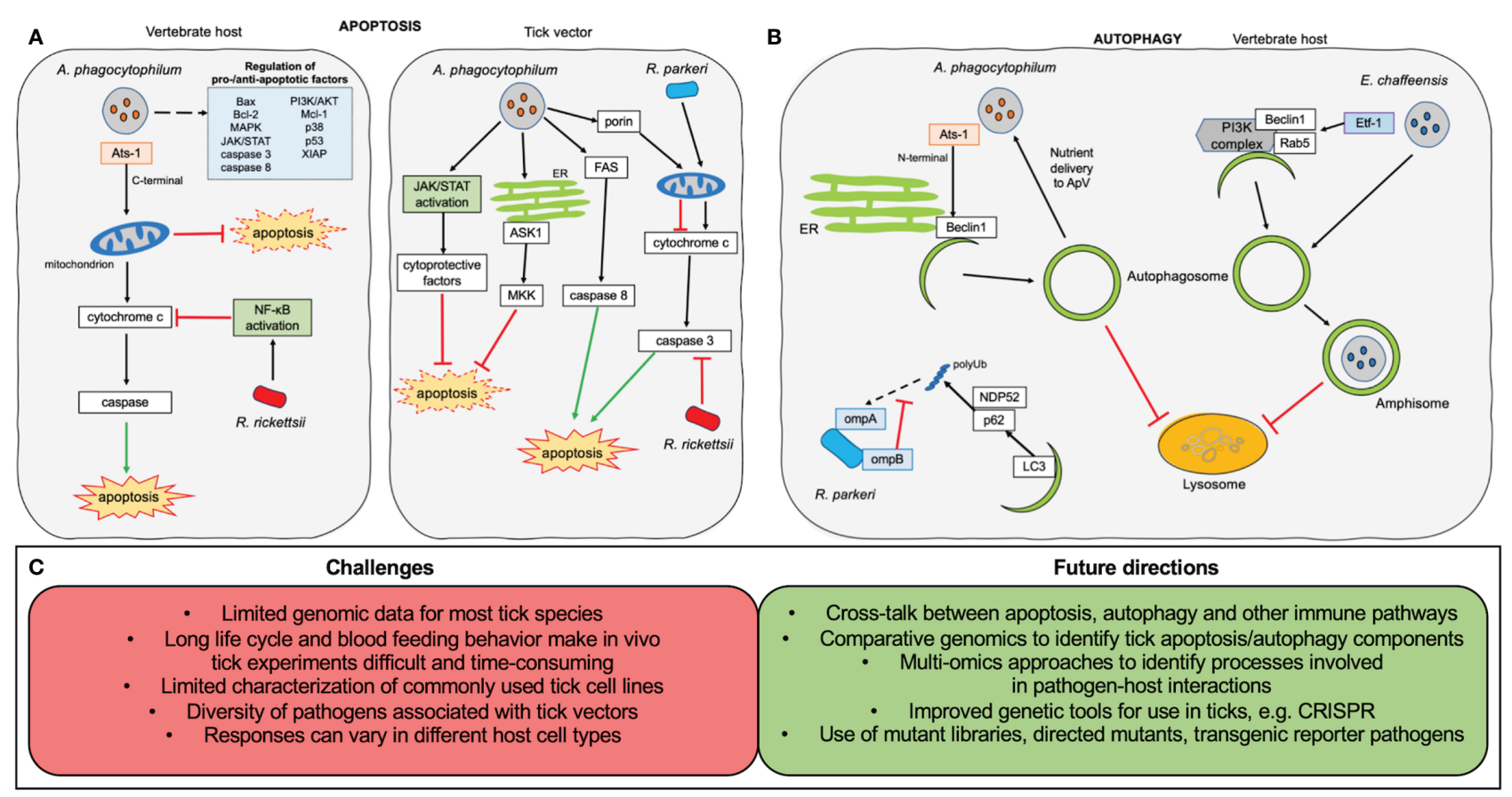

FIGURE 2 | Overview of apoptosis and autophagy research on tick-borne obligate intracellular pathogens. (A) Manipulation of apoptosis. In the vertebrate host (left) the C-terminal fragment of the Anaplasma phagocytophilum effector Ats-1 localizes to the host mitochondria where it inhibits apoptosis. A large number of kinases and pro-/anti-apoptotic factors (shown in pale blue box) also show changes in expression during A. phagocytophilum infection. Rickettsia rickettsii infection leads to NF-kB activation, inhibiting cytochrome c release from mitochondria resulting in downstream inhibition of apoptosis. In the tick vector (right), A. phagocytophilum uses multiple pathways to inhibit apoptosis, including activation of the JAK/STAT pathway, down-regulation of mitogen-activated protein kinase (MKK) and apoptosis signal-regulating kinase 1 (ASK1), decreasing FAS expression, and reducing porin expression to inhibit cytochrome c release from the mitochondria. Rickettsia rickettsii prevents caspase 3 activation in order to inhibit apoptosis. Rickettsia parkeri infection is associated with increased mitochondrial cytochrome $\mathrm{c}$ release, leading to increased activation of apoptosis, which is essential to its infection of tick cells. (B) Manipulation of autophagy. Experimental evidence only exists from mammalian systems. The $\mathrm{N}$-terminal portion of the A. phagocytophilum effector Ats-1 localizes to the endoplasmic reticulum (ER) where it interacts with Beclin1 to initiate autophagosome formation. Autophagosomes are prevented from trafficking to the lysosome and instead fuse with the bacterial vacuole to deliver membrane and nutrients to A. phagocytophilum. The Etf-1 effector secreted by Erhlichia chaffeensis interacts with Beclin1, Rab5 and the PI3K complex to induce autophagosome formation. The autophagosomes fuse with the Erhlichia-containing vacuole and are prevented from fusing with the lysosome by bacterial interference with the Wnt signalling pathway. During R. parkeri infection of macrophages, ompB shields the rickettsial surface protein ompA from polyubiquitination, preventing its recognition by the autophagy adaptors p62 and NDP52. (C) Challenges and future directions in the research of interactions of intracellular tick-borne pathogens with apoptosis and autophagy pathways.

random mutant libraries, the underlying mechanisms used by pathogens to influence autophagy and apoptosis pathways could be revealed, as well as identification of specific factors essential for bacterial invasion and replication in their tick vectors (Figure 2).

\section{SUMMARY}

Characterization of the involvement of the tick PCD machinery in pathogen acquisition, persistence, and transmission would help explain the natural cycle of tick-borne pathogens, as well as lead to the design of specific targets for new vaccines and drugs to prevent or treat TBDs. Greater knowledge of tick-borne intracellular pathogen and host (both ticks and mammals) interplay could have implications for understanding how the innate immune system contributes to the vector competence of various tick species for different intracellular pathogens and to the ability of vertebrate hosts to act as reservoirs or succumb to disease.

\section{AUTHOR CONTRIBUTIONS}

X-RW and BC conceived and wrote the article. All authors contributed to the article and approved the submitted version.

\section{FUNDING}

The study was financially supported by a grant to UGM from the NIH (2R01AI049424), and a grant to UGM from the Minnesota Agricultural Experiment Station (MIN-17-078).

\section{ACKNOWLEDGMENTS}

We thank Ulrike G. Munderloh for her insightful comments and revisions on this paper. 


\section{REFERENCES}

Alberdi, P., Espinosa, P. J., Cabezas-Cruz, A., and de la Fuente, J. (2016a). Anaplasma Phagocytophilum Manipulates Host Cell Apoptosis by Different Mechanisms to Establish Infection. Vet. Sci. 3(3), 15. doi: $10.3390 /$ vetsci3030015

Alberdi, P., Mansfield, K. L., Manzano-Román, R., Cook, C., Ayllón, N., Villar, M., et al. (2016b). Tissue-Specific Signatures in the Transcriptional Response to Anaplasma Phagocytophilum Infection of Ixodes Scapularis and Ixodes Ricinus Tick Cell Lines. Front. Cell. Infect. Microbiol. 6, 15. doi: 10.3389/ fcimb.2016.00020

Ameisen, J. C. (2002). On the Origin, Evolution, and Nature of Programmed Cell Death: A Timeline of Four Billion Years. Cell Death Differ. 9, 367-393. doi: $10.1038 /$ sj.cdd. 4400950

Ayllón, N., Villar, M., Busby, A. T., Kocan, K. M., Blouin, E. F., BonzónKulichenko, E., et al. (2013). Anaplasma Phagocytophilum Inhibits Apoptosis and Promotes Cytoskeleton Rearrangement for Infection of Tick Cells. Infect. Immun. 81, 2415-2425. doi: 10.1128/IAI.00194-13

Ayllón, N., Villar, M., Galindo, R. C., Kocan, K. M., Síma, R., López, J. A., et al. (2015). Systems Biology of Tissue-Specific Response to Anaplasma Phagocytophilum Reveals Differentiated Apoptosis in the Tick Vector Ixodes Scapularis. PloS Genet. 11, e1005120. doi: 10.1371/journal.pgen.1005120

Bechelli, J., Rumfield, C. S., Walker, D. H., Widen, S., Khanipov, K., and Fang, R. (2021). Subversion of Host Innate Immunity by Rickettsia Australis via a Modified Autophagic Response in Macrophages. Front. Immunol. 12, 638469. doi: 10.3389/fimmu.2021.638469

Bechelli, J., Vergara, L., Smalley, C., Buzhdygan, T. P., Bender, S., Zhang, W., et al. (2019). Atg5 Supports Rickettsia Australis Infection in Macrophages In Vitro and In Vivo. Infect. Immun. 87, e00651-18. doi: 10.1128/IAI.00651-18

Behar, S. M., and Briken, V. (2019). Apoptosis Inhibition by Intracellular Bacteria and its Consequence on Host Immunity. Curr. Opin. Immunol. 60, 103-110. doi: 10.1016/j.coi.2019.05.007

Bell-Sakyi, L. (1991). Continuous Cell Lines From the Tick Hyalomma Anatolicum Anatolicum. J. Parasitol. 77, 1006-1008. doi: 10.2307/3282757

Bell-Sakyi, L., Růžek, D., and Gould, E. A. (2009). Cell Lines From the Soft Tick Ornithodoros Moubata. Exp. Appl. Acarol. 49, 209-219. doi: 10.1007/s10493009-9258-y

Billmyre, R. B., Calo, S., Feretzaki, M., Wang, X., and Heitman, J. (2013). RNAi Function, Diversity, and Loss in the Fungal Kingdom. Chromosome Res. 21 (67), 561-572. doi: 10.1007/S10577-013-9388-2

Borjesson, D. L., Kobayashi, S. D., Whitney, A. R., Voyich, J. M., Argue, C. M., and DeLeo, F. R. (2005). Insights Into Pathogen Immune Evasion Mechanisms: Anaplasma Phagocytophilum Fails to Induce an Apoptosis Differentiation Program in Human Neutrophils. J. Immunol. 174, 6364-6372. doi: 10.4049/ jimmunol.174.10.6364

Bowman, A. S., and Sauer, J. R. (2004). Tick Salivary Glands: Function, Physiology and Future. Parasitology 129 (Suppl), S67-S81. doi: 10.1017/ s0031182004006468

Brossard, M., and Wikel, S. K. (2004). Tick Immunobiology. Parasitology 129, S161-S176. doi: 10.1017/S0031182004004834

Buchon, N., Silverman, N., and Cherry, S. (2014). Immunity in Drosophila Melanogaster-From Microbial Recognition to Whole-Organism Physiology. Nat. Rev. Immunol. 14, 796-810. doi: 10.1038/nri3763

Capelli-Peixoto, J., Carvalho, D. D., Johnson, W. C., Scoles, G. A., Fogaça, A. C., Daffre, S., et al. (2017). The Transcription Factor Relish Controls Anaplasma Marginale Infection in the Bovine Tick Rhipicephalus Microplus. Dev. Comp. Immunol. 74, 32-39. doi: 10.1016/j.dci.2017.04.005

CDC. (2018). Tickborne Diseases of the United States. A Reference Manual for Health Care Providers, Fifth Edition. Available at: https://www.cdc.gov/ticks/ tickbornediseases/TickborneDiseases-P.pdf.

Cervantes, J., Nagata, T., Uchijima, M., Shibata, K., and Koide, Y. (2007). Intracytosolic Listeria Monocytogenes Induces Cell Death Through Caspase-1 Activation in Murine Macrophages. Cell. Microbiol. 10, 41-52. doi: 10.1111/ j.1462-5822.2007.01012.x

Choi, K. S., Park, J. T., and Dumler, J. S. (2005). Anaplasma Phagocytophilum Delay of Neutrophil Apoptosis Through the P38 Mitogen-Activated Protein Kinase Signal Pathway. Infect. Immun. 73, 8209-8218. doi: 10.1128/ IAI.73.12.8209-8218.2005
Chowdhury, A., Modahl, C. M., Tan, S. T., Wei Xiang, B. W., Missé, D., Vial, T., et al. (2020). JNK Pathway Restricts DENV2, ZIKV and CHIKV Infection by Activating Complement and Apoptosis in Mosquito Salivary Glands. PloS Pathog. 16, e1008754. doi: 10.1371/journal.ppat.1008754

Chu, X., Zhang, B., Koeken, V. A. C. M., Gupta, M. K., and Li, Y. (2021). MultiOmics Approaches in Immunological Research. Front. Immunol. 12, 668045. doi: 10.3389/FIMMU.2021.668045

Clarke, T. E., and Clem, R. J. (2003). Insect Defenses Against Virus Infection: The Role of Apoptosis. Int. Rev. Immunol. 22, 401-424. doi: 10.1080/ 08830180305215

Clifton, D. R., Goss, R. A., Sahni, S. K., Van Antwerp, D., Baggs, R. B., Marder, V. J., et al. (1998). NF-KB-Dependent Inhibition of Apoptosis is Essential for Host Cell Survival During Rickettsia Rickettsii Infection. Proc. Natl. Acad. Sci. U. S. A. 95, 4646-4651. doi: 10.1073/pnas.95.8.4646

Cramaro, W. J., Hunewald, O. E., Bell-Sakyi, L., and Muller, C. P. (2017). Genome Scaffolding and Annotation for the Pathogen Vector Ixodes Ricinus by UltraLong Single Molecule Sequencing. Parasit. Vectors 10, 71. doi: 10.1186/s13071017-2008-9

Durvasula, R. V., Gumbs, A., Panackal, A., Kruglov, O., Aksoy, S., Merrifield, R. B., et al. (1997). Prevention of Insect-Borne Disease: An Approach Using Transgenic Symbiotic Bacteria. Proc. Natl. Acad. Sci. U. S. A. 94, 3274-3278. doi: $10.1073 /$ pnas.94.7.3274

Elmore, S. (2007). Apoptosis: A Review of Programmed Cell Death. Toxicol. Pathol. 35, 495-516. doi: 10.1080/01926230701320337

Engström, P., Burke, T. P., Mitchell, G., Ingabire, N., Mark, K. G., Golovkine, G., et al. (2019). Evasion of Autophagy Mediated by Rickettsia Surface Protein OmpB is Critical for Virulence. Nat. Microbiol. 4, 2538-2551. doi: 10.1038/ s41564-019-0583-6

Everett, H., and McFadden, G. (1999). Apoptosis: An Innate Immune Response to Virus Infection. Trends Microbiol. 7, 160-165. doi: 10.1016/S0966-842X(99) $01487-0$

Fairlie, W. D., Tran, S., and Lee, E. F. (2020). "Crosstalk Between Apoptosis and Autophagy Signaling Pathways," in International Review of Cell and Molecular Biology (Amsterdam: Academic Press), 115-158. doi: 10.1016/ bs.ircmb.2020.01.003

Feng, Y., He, D., Yao, Z., and Klionsky, D. J. (2014). The Machinery of Macroautophagy. Cell Res. 24, 24-41. doi: 10.1038/cr.2013.168

Flegel, T. W. (2007). Update on Viral Accommodation, a Model for Host-Viral Interaction in Shrimp and Other Arthropods. Dev. Comp. Immunol. 31, 217231. doi: 10.1016/j.dci.2006.06.009

Flores Fernández, J. M., Barragán Álvarez, C. P., Sánchez Hernández, C. V., Padilla Camberos, E., González Castillo, C., Ortunõ Sahagún, D., et al. (2016). Molecular Characterization and Expression Analysis of Three Novel Autophagy-Related Genes From the Cattle Tick Rhipicephalus (Boophilus) Microplus (Acari: Ixodidae). Parasitology 143, 1802-1809. doi: 10.1017/ S0031182016001542

Flores Fernández, J. M., Gutiérrez Ortega, A., Rosario Cruz, R., Padilla Camberos, E., Álvarez, Á. H., and Martínez Velázquez, M. (2014). Molecular Cloning and Characterization of Two Novel Autophagy-Related Genes Belonging to the ATG8 Family From the Cattle Tick Rhipicephalus (Boophilus) Microplus (Acari: Ixodidae). Exp. Appl. Acarol. 64, 533-542. doi: 10.1007/s10493-014-9838-3

Fogaça, A. C., Sousa, G., Pavanelo, D. B., Esteves, E., Martins, L. A., Urbanová, V., et al. (2021). Tick Immune System: What Is Known, the Interconnections, the Gaps, and the Challenges. Front. Immunol. 12, 628054. doi: 10.3389/ fimmu.2021.628054

Fuchs, Y., and Steller, H. (2011). Programmed Cell Death in Animal Development and Disease. Cell 147, 742-758. doi: 10.1016/j.cell.2011.10.033

Fuller, J., Surtees, R. A., Shaw, A. B., Álvarez-Rodríguez, B., Slack, G. S., Bell-Sakyi, L., et al. (2019). Hazara Nairovirus Elicits Differential Induction of Apoptosis and Nucleocapsid Protein Cleavage in Mammalian and Tick Cells. J. Gen. Virol. 100, 392-402. doi: 10.1099/jgv.0.001211

Ge, Y., and Rikihisa, Y. (2006). Anaplasma Phagocytophilum Delays Spontaneous Human Neutrophil Apoptosis by Modulation of Multiple Apoptotic Pathways. Cell. Microbiol. 8, 1406-1416. doi: 10.1111/j.1462-5822.2006.00720.x

Ghosh, S., Azhahianambi, P., and Yadav, M. P. (2007). Upcoming and Future Strategies of Tick Control: A Review. J. Vector Borne Dis. 44, 79-89.

González Castillo, C., Ortuño Sahagún, D., and Martínez Velázquez, M. (2019). Expression Dynamics of Autophagy-Related Genes in the Cattle Tick 
Rhipicephalus Microplus During Embryonic Development and Under Increasing Larval Starvation. Exp. Appl. Acarol. 79, 255-266. doi: 10.1007/ s10493-019-00428-7

Gray, S. M., and Banerjee, N. (1999). Mechanisms of Arthropod Transmission of Plant and Animal Viruses. Microbiol. Mol. Biol. Rev. 63, 128-148. doi: 10.1128/ MMBR.63.1.128-148.1999

Gray, J. S., Dautel, H., Estrada-Peña, A., Kahl, O., and Lindgren, E. (2009). Effects of Climate Change on Ticks and Tick-Borne Diseases in Europe. Interdiscip. Perspect. Infect. Dis. 2009, 593232. doi: 10.1155/2009/593232

Green, D. R., and Kroemer, G. (2004). The Pathophysiology of Mitochondrial Cell Death. Science 305, 626-629. doi: 10.1126/science.1099320

Gulia-Nuss, M., Nuss, A. B., Meyer, J. M., Sonenshine, D. E., Roe, M. R., Waterhouse, R. M., et al. (2016). Genomic Insights Into the Ixodes Scapularis Tick Vector of Lyme Disease. Nat. Commun. 7, 10507. doi: 10.1038/ncomms10507

Hart, C. E., and Thangamani, S. (2021). Tick-Virus Interactions: Current Understanding and Future Perspectives. Parasite Immunol. 43, e12815. doi: $10.1111 /$ pim.12815

Hasin, Y., Seldin, M., and Lusis, A. (2017). Multi-Omics Approaches to Disease. Genome Biol. 18, 83. doi: 10.1186/s13059-017-1215-1

Hua, F., Li, K., Shang, S., Wang, F., and Hu, Z. (2019). "Immune Signaling and Autophagy Regulation," in Advances in Experimental Medicine and Biology (Singapore: Springer), 551-593. doi: 10.1007/978-981-15-0602-4_26

Jacobson, M. D., Weil, M., and Raff, M. C. (1997). Programmed Cell Death in Animal Development. Cell 88, 347-354. doi: 10.1016/S0092-8674(00)81873-5

Jia, N., Wang, J., Shi, W., Du, L., Sun, Y., Zhan, W., et al. (2020). Large-Scale Comparative Analyses of Tick Genomes Elucidate Their Genetic Diversity and Vector Capacities. Cell 182, 1328-1340.e13. doi: 10.1016/j.cell.2020.07.023

Jongejan, F., and Uilenberg, G. (2004). The Global Importance of Ticks. Parasitology 129 (Suppl), S3-14. doi: 10.1017/s0031182004005967

Kader, M., Alaoui-EL-Azher, M., Vorhauer, J., Kode, B. B., Wells, J. Z., Stolz, D., et al. (2017). MyD88-Dependent Inflammasome Activation and Autophagy Inhibition Contributes to Ehrlichia-Induced Liver Injury and Toxic Shock. PloS Pathog. 13, e1006644. doi: 10.1371/journal.ppat.1006644

Kawano, S., Umemiya-Shirafuji, R., Boldbaatar, D., Matsuoka, K., Tanaka, T., and Fujisaki, K. (2011). Cloning and Characterization of the Autophagy-Related Gene 6 From the Hard Tick, Haemaphysalis Longicornis. Parasitol. Res. 109, 1341-1349. doi: 10.1007/s00436-011-2429-x

Koonin, E. V., and Krupovic, M. (2019). Origin of Programmed Cell Death From Antiviral Defense? Proc. Natl. Acad. Sci. 116, 16167-16169. doi: 10.1073/ pnas. 1910303116

Kurokawa, C., Lynn, G. E., Pedra, J. H. F., Pal, U., Narasimhan, S., and Fikrig, E. (2020). Interactions Between Borrelia Burgdorferi and Ticks. Nat. Rev. Microbiol. 18, 587-600. doi: 10.1038/s41579-020-0400-5

Labbé, K., and Saleh, M. (2008). Cell Death in the Host Response to Infection. Cell Death Differ. 15, 1339-1349. doi: 10.1038/cdd.2008.91

L'Amoreaux, W. J., Junaid, L., and Trevidi, S. (2003). Morphological Evidence That Salivary Gland Degeneration in the American Dog Tick, Dermacentor Variabilis (Say), Involves Programmed Cell Death. Tissue Cell 35, 95-99. doi: 10.1016/S0040-8166(02)00109-X

Lehman, S. S., Noriea, N. F., Aistleitner, K., Clark, T. R., Dooley, C. A., Nair, V., et al. (2018). The Rickettsial Ankyrin Repeat Protein 2 Is a Type IV Secreted Effector That Associates With the Endoplasmic Reticulum. MBio 9, e00975-18. doi: $10.1128 / \mathrm{mBio} .00975-18$

Levine, B., and Deretic, V. (2007). Unveiling the Roles of Autophagy in Innate and Adaptive Immunity. Nat. Rev. Immunol. 7, 767-777. doi: 10.1038/nri2161

Lina, T. T., Luo, T., Velayutham, T. S., Das, S., and McBride, J. W. (2017). Ehrlichia Activation of Wnt-PI3K-mTOR Signaling Inhibits Autolysosome Generation and Autophagic Destruction by the Mononuclear Phagocyte. Infect. Immun. 85, e00690-17. doi: 10.1128/IAI.00690-17

Lin, M., Liu, H., Xiong, Q., Niu, H., Cheng, Z., Yamamoto, A., et al. (2016). Ehrlichia Secretes Etf-1 to Induce Autophagy and Capture Nutrients for Its Growth Through RAB5 and Class III Phosphatidylinositol 3-Kinase. Autophagy 12, 2145-2166. doi: 10.1080/15548627.2016.1217369

Liu, H., Bao, W., Lin, M., Niu, H., and Rikihisa, Y. (2012). Ehrlichia Type IV Secretion Effector ECH0825 Is Translocated to Mitochondria and Curbs ROS and Apoptosis by Upregulating Host MnSOD. Cell. Microbiol. 14, 1037-1050. doi: $10.1111 / j .1462-5822.2012 .01775 . x$
Ma, Z., Li, R., Hu, R., Zheng, W., Yu, S., Cheng, K., et al. (2021). Anaplasma Phagocytophilum AptA Enhances the UPS, Autophagy, and Anti-Apoptosis of Host Cells by PSMG3. Int. J. Biol. Macromol. 184, 497-508. doi: 10.1016/ j.ijbiomac.2021.06.039

Mansfield, K. L., Cook, C., Ellis, R. J., Bell-Sakyi, L., Johnson, N., Alberdi, P., et al. (2017). Tick-Borne Pathogens Induce Differential Expression of Genes Promoting Cell Survival and Host Resistance in Ixodes Ricinus Cells. Parasit. Vectors 10, 81. doi: 10.1186/s13071-017-2011-1

Mao, H., and Kaufman, W. R. (1999). Profile of the Ecdysteroid Hormone and its Receptor in the Salivary Gland of the Adult Female Tick, Amblyomma Hebraeum. Insect Biochem. Mol. Biol. 29, 33-42. doi: 10.1016/S0965-1748 (98)00102-7

Mao, H., McBlain, W. A., and Kaufman, W. R. (1995). Some Properties of the Ecdysteroid Receptor in the Salivary Gland of the Ixodid Tick, Amblyomma Hebraeum. Gen. Comp. Endocrinol. 99, 340-348. doi: 10.1006/gcen.1995.1118

Martin, C. J., Booty, M. G., Rosebrock, T. R., Nunes-Alves, C., Desjardins, D. M., Keren, I., et al. (2012). Efferocytosis Is an Innate Antibacterial Mechanism. Cell Host Microbe 12, 289-300. doi: 10.1016/j.chom.2012.06.010

Martins, L. A., Palmisano, G., Cortez, M., Kawahara, R., de Freitas Balanco, J. M., Fujita, A., et al. (2020). The Intracellular Bacterium Rickettsia Rickettsii Exerts an Inhibitory Effect on the Apoptosis of Tick Cells. Parasit. Vectors 13, 603. doi: 10.1186/s13071-020-04477-5

Mattila, J. T., Burkhardt, N. Y., Hutcheson, H. J., Munderloh, U. G., and Kurtti, T. J. (2007). Isolation of Cell Lines and a Rickettsial Endosymbiont From the Soft Tick Carios Capensis (Acari: Argasidae: Ornithodorinae). J. Med. Entomol. 44, 1091-1101. doi: 10.1093/jmedent/44.6.1091

McClure Carroll, E. E., Wang, X., Shaw, D. K., O’Neal, A. J., Oliva Chávez, A. S., Brown, L. J., et al. (2019). P47 Licenses Activation of the Immune Deficiency Pathway in the Tick Ixodes Scapularis. Proc. Natl. Acad. Sci. 116, 205-210. doi: 10.1073/pnas.1808905116

Menze, M. A., Fortner, G., Nag, S., and Hand, S. C. (2010). Mechanisms of Apoptosis in Crustacea: What Conditions Induce Versus Suppress Cell Death? Apoptosis 15, 293-312. doi: 10.1007/s10495-009-0443-6

Moura-Martiniano, N. O., Machado-Ferreira, E., Gazêta, G. S., and Soares, C. A. G. (2017). Relative Transcription of Autophagy-Related Genes in Amblyomma Sculptum and Rhipicephalus Microplus Ticks. Exp. Appl. Acarol. 73, 401-428. doi: 10.1007/s10493-017-0193-z

Munderloh, U. G., and Kurtti, T. J. (1989). Formulation of Medium for Tick Cell Culture. Exp. Appl. Acarol. 7, 219-229. doi: 10.1007/BF01194061

Niu, H., Kozjak-Pavlovic, V., Rudel, T., and Rikihisa, Y. (2010). Anaplasma Phagocytophilum Ats-1 Is Imported Into Host Cell Mitochondria and Interferes With Apoptosis Induction. PloS Pathog. 6, e1000774. doi: 10.1371/ journal.ppat.1000774

Niu, H., Xiong, Q., Yamamoto, A., Hayashi-Nishino, M., and Rikihisa, Y. (2012). Autophagosomes Induced by a Bacterial Beclin 1 Binding Protein Facilitate Obligatory Intracellular Infection. Proc. Natl. Acad. Sci. U. S. A. 109, 2080020807. doi: 10.1073/pnas.1218674109

Niu, H., Yamaguchi, M., and Rikihisa, Y. (2008). Subversion of Cellular Autophagy by Anaplasma Phagocytophilum. Cell. Microbiol. 10, 593-605. doi: 10.1111/j.1462-5822.2007.01068.x

Nunes, E. T., Bechara, G. H., Saito, K. C., Denardi, S. E., Oliveira, P. R., and Mathias, M. I. C. (2005). Morphological, Histological, and Ultrastructural Characterization of Degenerating Salivary Glands in Females of the Cattle-Tick Rhipicephalus (Boophilus) Microplus (CANESTRINI 1887) (Acari: Ixodidae). Micron 36, 437-447. doi: 10.1016/j.micron.2005.03.010

Patterson, L. L., Byerly, C. D., and McBride, J. W. (2021). Anaplasmataceae: Dichotomous Autophagic Interplay for Infection. Front. Immunol. 12. doi: $10.3389 /$ fimmu.2021.642771

Ramphul, U. N., Garver, L. S., Molina-Cruz, A., Canepa, G. E., and Barillas-Mury, C. (2015). Plasmodium Falciparum Evades Mosquito Immunity by Disrupting JNKMediated Apoptosis of Invaded Midgut Cells. Proc. Natl. Acad. Sci. U. S. A. 112, 1273-1280. doi: 10.1073/pnas.1423586112

Riedl, S. J., and Salvesen, G. S. (2007). The Apoptosome: Signalling Platform of Cell Death. Nat. Rev. Mol. Cell Biol. 8, 405-413. doi: 10.1038/nrm2153

Romanelli, D., Casati, B., Franzetti, E., and Tettamanti, G. (2014). A Molecular View of Autophagy in Lepidoptera. BioMed. Res. Int. 2014, 1-11. doi: 10.1155/ 2014/902315 
Rosendale, A. J., Dunlevy, M. E., McCue, M. D., and Benoit, J. B. (2019). Progressive Behavioural, Physiological and Transcriptomic Shifts Over the Course of Prolonged Starvation in Ticks. Mol. Ecol. 28, 49-65. doi: 10.1111/mec.14949

Rudel, T., Kepp, O., and Kozjak-Pavlovic, V. (2010). Interactions Between Bacterial Pathogens and Mitochondrial Cell Death Pathways. Nat. Rev. Microbiol. 8, 693-705. doi: 10.1038/nrmicro2421

Sahni, A., Narra, H. P., and Sahni, S. K. (2020). Activation of Mechanistic Target of Rapamycin (mTOR) in Human Endothelial Cells Infected With Pathogenic Spotted Fever Group Rickettsiae. Int. J. Mol. Sci. 21, 7179. doi: 10.3390/ ijms21197179

Salje, J. (2021). Cells Within Cells: Rickettsiales and the Obligate Intracellular Bacterial Lifestyle. Nat. Rev. Microbiol. 19, 375-390. doi: 10.1038/s41579-02000507-2

Scaife, H., Woldehiwet, Z., Hart, C. A., and Edwards, S. W. (2003). Anaplasma Phagocytophilum Reduces Neutrophil Apoptosis In Vivo. Infect. Immun. 71, 1995-2001. doi: 10.1128/IAI.71.4.1995-2001.2003

Scopinho Furquim, K. C., Bechara, G. H., and Camargo Mathias, M. I. (2008). Death by Apoptosis in Salivary Glands of Females of the Tick Rhipicephalus Sanguineus (Latreille 1806) (Acari: Ixodidae). Exp. Parasitol. 119, 152-163. doi: 10.1016/j.exppara.2008.01.021

Shaw, D. K., Wang, X., Brown, L. J., Chávez, A. S. O., Reif, K. E., Smith, A. A., et al. (2017). Infection-Derived Lipids Elicit an Immune Deficiency Circuit in Arthropods. Nat. Commun. 8, 14401. doi: 10.1038/ncomms14401

Sokolova, I. M. (2009). Apoptosis in Molluscan Immune Defense. Invertebr. Surviv. J. 6, 49-58.

Sonenshine, D., and Roe, R. M. (2013). Biology of Ticks, 2nd ed, (New York: Oxford University Press).

Taatjes, D. J., Sobel, B. E., and Budd, R. C. (2008). Morphological and Cytochemical Determination of Cell Death by Apoptosis. Histochem. Cell Biol. 129, 33-43. doi: 10.1007/s00418-007-0356-9

Tafesh-Edwards, G., and Eleftherianos, I. (2020). JNK Signaling in Drosophila Immunity and Homeostasis. Immunol. Lett. 226, 7-11. doi: 10.1016/ j.imlet.2020.06.017

Taylor, R. C., Cullen, S. P., and Martin, S. J. (2008). Apoptosis: Controlled Demolition at the Cellular Level. Nat. Rev. Mol. Cell Biol. 9, 231-241. doi: $10.1038 / \mathrm{nrm} 2312$

Tominello, T. R., Oliveira, E. R. A., Hussain, S. S., Elfert, A., Wells, J., Golden, B., et al. (2019). Emerging Roles of Autophagy and Inflammasome in Ehrlichiosis. Front. Immunol. 10. doi: 10.3389/FIMMU.2019.01011

Uchiyama, T., Kishi, M., and Ogawa, M. (2012). Restriction of the Growth of a Nonpathogenic Spotted Fever Group Rickettsia. FEMS Immunol. Med. Microbiol. 64, 42-47. doi: 10.1111/j.1574-695X.2011.00879.x

Umemiya, R., Matsuo, T., Hatta, T., Sakakibara, S., Boldbaatar, D., and Fujisaki, K. (2007). Cloning and Characterization of an Autophagy-Related Gene, ATG12, From the Three-Host Tick Haemaphysalis Longicornis. Insect Biochem. Mol. Biol. 37, 975-984. doi: 10.1016/j.ibmb.2007.05.006

Umemiya-Shirafuji, R., Galay, R. L., Maeda, H., Kawano, S., Tanaka, T., Fukumoto, S., et al. (2014). Expression Analysis of Autophagy-Related Genes in the Hard Tick Haemaphysalis Longicornis. Vet. Parasitol. 201, 169-175. doi: 10.1016/j.vetpar.2014.01.024

Umemiya-Shirafuji, R., Matsuo, T., Liao, M., Boldbaatar, D., Battur, B., Suzuki, H., et al. (2010). Increased Expression of ATG Genes During Nonfeeding Periods in the Tick Haemaphysalis Longicornis. Autophagy 6, 473-481. doi: 10.4161/ auto.6.4.11668

Van Opdenbosch, N., and Lamkanfi, M. (2019). Caspases in Cell Death, Inflammation, and Disease. Immunity 50, 1352-1364. doi: 10.1016/ j.immuni.2019.05.020
Villar, M., Ayllón, N., Alberdi, P., Moreno, A., Moreno, M., Tobes, R., et al. (2015) Integrated Metabolomics, Transcriptomics and Proteomics Identifies Metabolic Pathways Affected by Anaplasma Phagocytophilum Infection in Tick Cells. Mol. Cell. Proteomics 14, 3154-3172. doi: 10.1074/mcp.M115.051938

Vo, P. L. H., Ronda, C., Klompe, S. E., Chen, E. E., Acree, C., Wang, H. H., et al. (2021). CRISPR RNA-Guided Integrases for High-Efficiency, Multiplexed Bacterial Genome Engineering. Nat. Biotechnol. 39, 480-489. doi: 10.1038/ s41587-020-00745-y

Voss, O. H., and Rahman, M. S. (2021). Rickettsia-Host Interaction: Strategies of Intracytosolic Host Colonization. Pathog. Dis. 79, ftab015. doi: 10.1093/ femspd/ftab015

Wang, X.-R., Burkhardt, N. Y., Kurtti, T. J., Oliver, J. D., Price, L. D., Cull, B., et al. (2021). Mitochondrion-Dependent Apoptosis Is Essential for Rickettsia Parkeri Infection and Replication in Vector Cells. mSystems 6, e01209-20. doi: 10.1128/mSystems.01209-20

Wang, X.-R., Kurtti, T. J., Oliver, J. D., and Munderloh, U. G. (2020). The Identification of Tick Autophagy-Related Genes in Ixodes Scapularis Responding to Amino Acid Starvation. Ticks Tick Borne Dis. 11, 101402. doi: 10.1016/j.ttbdis.2020.101402

Wang, Z., and Li, C. (2020). Xenophagy in Innate Immunity: A Battle Between Host and Pathogen. Dev. Comp. Immunol. 109, 103693. doi: 10.1016/ j.dci.2020.103693

Wang, Y., Zhang, H., Luo, L., Zhou, Y., Cao, J., Xuan, X., et al. (2021). ATG5 is Instrumental in the Transition From Autophagy to Apoptosis During the Degeneration of Tick Salivary Glands. PloS Negl. Trop. Dis. 15, e0009074. doi: 10.1371/journal.pntd.0009074

Wang, H., Zhang, X., Wang, X., Zhang, B., Wang, M., Yang, X., et al. (2018). Comprehensive Analysis of the Global Protein Changes That Occur During Salivary Gland Degeneration in Female Ixodid Ticks Haemaphysalis Longicornis. Front. Physiol. 9, 1943. doi: 10.3389/fphys.2018.01943

Wilke, A. B. B., and Marrelli, M. T. (2015). Paratransgenesis: A Promising New Strategy for Mosquito Vector Control. Parasit. Vectors 8, 342. doi: 10.1186/ s13071-015-0959-2

Willingham, S. B., Bergstralh, D. T., O'Connor, W., Morrison, A. C., Taxman, D. J., Duncan, J. A., et al. (2007). Microbial Pathogen-Induced Necrotic Cell Death Mediated by the Inflammasome Components CIAS1/Cryopyrin/NLRP3 and ASC. Cell Host Microbe 2, 147-159. doi: 10.1016/j.chom.2007.07.009

Yu, X., Zhou, Y., Cao, J., Zhang, H., Gong, H., and Zhou, J. (2017). Caspase-1 Participates in Apoptosis of Salivary Glands in Rhipicephalus Haemaphysaloides. Parasit. Vectors 10, 225. doi: 10.1186/s13071-017-2161-1

Conflict of Interest: The authors declare that the research was conducted in the absence of any commercial or financial relationships that could be construed as a potential conflict of interest.

Publisher's Note: All claims expressed in this article are solely those of the authors and do not necessarily represent those of their affiliated organizations, or those of the publisher, the editors and the reviewers. Any product that may be evaluated in this article, or claim that may be made by its manufacturer, is not guaranteed or endorsed by the publisher.

Copyright $(2022$ Wang and Cull. This is an open-access article distributed under the terms of the Creative Commons Attribution License (CC BY). The use, distribution or reproduction in other forums is permitted, provided the original author(s) and the copyright owner(s) are credited and that the original publication in this journal is cited, in accordance with accepted academic practice. No use, distribution or reproduction is permitted which does not comply with these terms. 